Rabaska

Revue d'ethnologie de l'Amérique française

BONNOT, THIERRY. L'Attachement aux choses. Paris, CNRS

Éditions, "Le passé recomposé ", 2014, 239 p. ISBN

978-2-271-08116-2

\title{
Laurence Provencher-St-Pierre
}

Volume 15, 2017

URI : https://id.erudit.org/iderudit/1041138ar

DOI : https://doi.org/10.7202/1041138ar

Aller au sommaire du numéro

Éditeur(s)

Société québécoise d'ethnologie

ISSN

1703-7433 (imprimé)

1916-7350 (numérique)

Découvrir la revue

Citer ce compte rendu

Provencher-St-Pierre, L. (2017). Compte rendu de [BONNOT, THIERRY.

L'Attachement aux choses. Paris, CNRS Éditions, « Le passé recomposé ", 2014,

239 p. ISBN 978-2-271-08116-2]. Rabaska, 15, 226-229.

https://doi.org/10.7202/1041138ar

Ce document est protégé par la loi sur le droit d'auteur. L'utilisation des services d'Érudit (y compris la reproduction) est assujettie à sa politique d'utilisation que vous pouvez consulter en ligne.

https://apropos.erudit.org/fr/usagers/politique-dutilisation/
Cet article est diffusé et préservé par Érudit.

Érudit est un consortium interuniversitaire sans but lucratif composé de l'Université de Montréal, l'Université Laval et l'Université du Québec à Montréal. Il a pour mission la promotion et la valorisation de la recherche. https://www.erudit.org/fr/ 
la crise du système de santé, l'enjeu de préservation du patrimoine est identitaire. Si, comme le relève Pauline Chevallier pour le Musée de l'Assistance Publique-Hôpitaux de Paris (AP-HP), les musées hospitaliers incarnent un pôle fédérateur dans un monde en mutation, ces espaces sont fragiles. Et pour cause, mentionnons depuis la publication de l'ouvrage, la vente de l'hôtel de Miramion qui hébergeait le musée de l'AP-HP.

Archives et musées : le théâtre du patrimoine (France-Canada) fait un tour d'horizon des réflexions et pratiques touchant aux archives, musées et établissements hospitaliers. Si la question de la patrimonialisation, déclinée en quatre chapitres (archives, patrimoine matériel, patrimoine immatériel, patrimoine religieux et médical) est structurée autour d'articles théoriques, la diversité des thématiques abordées et des auteurs assemblés intrigue et s'avère passionnante. L'ouvrage offre une pléiade de pistes de réflexion et confirme le dynamisme de la coopération en muséologie et en archivistique au Canada (principalement au Québec) et en France.

Violette Loget

Université du Québec à Montréal

Bonnot, Thierry. L'Attachement aux choses. Paris, CNRs Éditions, « Le passé recomposé », 2014, 239 p. ISBN 978-2-271-08116-2.

Douze ans après la parution de La Vie des objets : d'ustensiles banals à objets de collection (Paris, Maison des sciences de l'homme, 2002, 246 p.), l'anthropologue Thierry Bonnot publie un second ouvrage dans lequel il fait le point sur l'étude des objets en sciences humaines et sociales. Dans cet essai écrit à la première personne, Bonnot fait état des études passées et actuelles en culture matérielle, trace le récit de ses propres expériences sur le terrain et ouvre de nouvelles perspectives de recherche. L'ouvrage se compose de trois parties qui nous font découvrir de manière graduelle la vision de l'auteur et l'évolution de sa pensée au cours des dernières années. La position défendue par Bonnot est la suivante: «Les sciences sociales doivent passer d'une étude des objets comme sources d'un savoir sur les hommes en société, à une étude historiquement située des objets dans leur relation avec les hommes et dans leur influence sur les relations entre les hommes. » (p. 7.)

Dans la première partie, Bonnot dresse un portrait critique de ce domaine de recherche que représentent les études sur les objets en sciences sociales à partir d'un corpus d'auteurs choisis et issus de différents horizons disciplinaires. D'abord, il examine les nombreuses conceptions et définitions auxquelles renvoie la culture matérielle et fait état des débats et des limites entourant l'utilisation de cette expression. Renvoyant à un champ de 
recherche immense et hétérogène, les termes de culture matérielle doivent être employés avec parcimonie. Ensuite, Bonnot critique les aspirations des chercheurs dans ce domaine et analyse un certain nombre de dualismes (le matériel et l'immatériel; l'objet technique et l'objet social; l'objet et le sujet) qui ont jusqu'ici enfermé la recherche dans une impasse. Ce tour d'horizon des auteurs et des approches proposé en première partie permet à Bonnot de démontrer comment les sciences sociales avaient considéré les objets matériels en tant que sources fiables et de quelle manière les disciplines ont graduellement révélé la complexité du rapport aux choses, effaçant du même coup cette certitude. Il formule également une idée centrale de son argumentation, soit l'inutilité de chercher à élaborer une théorie universelle de la culture matérielle : «À chaque situation dans laquelle sont impliqués des sujets et des objets singuliers correspond un certain nombre d'enjeux sociaux et/ou politiques qui échappent par définition à toute théorie générale, que celle-ci porte exclusivement sur les pratiques, sur les techniques ou sur les représentations. » (p. 70.)

Dans la deuxième partie basée sur l'étude d'exemples tirés d'expériences artistiques ou de recherches empiriques, Bonnot aborde principalement trois thèmes. D'abord, il s'interroge sur la place du hasard dans les études en sciences sociales, se demandant si l'indéterminé doit être pris en compte dans les enquêtes. En s'appuyant sur la démarche de deux artistes qui ont abordé la notion de hasard quant à la présence (ou l'absence) de certains objets dans une pièce ou sur un meuble, Bonnot se prête lui-même à l'exercice. L'autoanalyse d'un espace de son intérieur domestique l'amène à affirmer que « les objets ne sont jamais là par hasard. » Il ajoute d'ailleurs que « c'est le rôle des sciences sociales de travailler à ce qui les a amenés [à cet endroit], à leur parcours, tributaire d'intentions et d'actions humaines. Dit autrement, c'est ce qui ne relève pas du hasard dans la présence d'un objet dans une situation donnée qui constitue l'objet des sciences sociales. » (p. 83) Ensuite, il s'intéresse à la question de l'objet en contexte muséal à partir d'un exemple de musée fictif. L'analyse de ce musée installé dans la maison d'un homme où serait présenté tous les témoins de sa vie personnelle, entraîne une réflexion sur la notion ambigüe d'objets de musée, voire plus largement sur celle d'objet patrimonial. Enfin, Bonnot poursuit cette réflexion et revient sur la manière dont l'objet (dans ce cas-ci muséal) a cessé d'être considéré comme une source incontestable. Le concept d'objet-témoin étant dépassé, l'objet ne peut plus aujourd'hui se limiter à représenter une seule réalité. Il ne renvoie pas qu'à une fonction, qu'à un lieu, qu'à une époque ou qu'à une population. Au contraire, il a une identité qui évolue dans le temps et l'espace. Dès lors, le second postulat soutenu par Bonnot devient clair : « Il ne convient pas 
ici d'assigner aux objets des significations mais de leur associer des actions inscrites dans un contexte local et un enchaînement historique. » (p. 118) Ainsi, comme il l'affirme par la suite, « [1]'objet serait dans ce cadre d'étude un nœud de relations sociales, et non pas un élément porteur de sens symbolique ou un témoin d'une structure sociale. » (p. 139)

En troisième partie, Bonnot approfondit sa réflexion en identifiant différentes pistes de recherches. Il propose d'abord une lecture plus nuancée de la notion de biographie d'objet d'Igor Kopytoff ${ }^{2}$. Puis, Bonnot démontre que le modèle théorique proposé par Krzysztof Pomian (Collectionneurs, amateurs et curieux. Paris, Venise XVI - XVIII siècle, Paris, Gallimard, 1987, 367 p.) est trop rigide et qu'il n'arrive pas à refléter la réalité beaucoup plus complexe de la patrimonialisation. Ensuite, après avoir étudié une série de courts exemples supplémentaires, Bonnot formule une série de pistes méthodologiques, conceptuelles, linguistiques et épistémologiques. Nous en retenons ici trois qui semblent particulièrement prometteuses.

La première proposition concerne l'importance du récit et de l'expérience du chercheur. Bonnot invite le chercheur à ne pas s'exclure du discours narratif construit à partir du travail d'enquête qui permet de retracer la trajectoire d'un objet. Il lui apparaît essentiel d'adopter une méthode réflexive qui tient compte de sa propre subjectivité, rappelant que le chercheur, en s'intéressant à un objet plutôt qu'à un autre, participe à l'histoire de cet objet. Ainsi, les subjectivités de l'ensemble des gens qui fournissent des informations sur un objet et celles de l'ethnologue qui l'étudie doivent être incluses dans le récit de l'expérience singulière que représente la rencontre avec cet objet étudié.

Ensuite, Bonnot introduit la notion de valence. Soulevant le problème que représente l'attribution d'une valeur (toujours relative) aux choses alors que celle-ci change selon les contextes et la perception des gens, il propose de remplacer la notion de valeur par celle de valence. Par exemple, dans le cas de l'étude d'un objet patrimonial, la valence « serait l'ensemble de ses liaisons avec des individus, la combinaison de son histoire singulière et collective, l'attraction ou la répulsion qu'il a suscitée et suscite encore, tout ce qui dans sa biographie a compté pour que cet objet soit conservé et devienne patrimonial. » (p. 188) Cette notion de valence apparaît tout à fait cohérente avec la position de l'auteur qui conçoit l'objet comme un « nœud de relation sociale ».

Enfin, il propose de favoriser à l'expression méthode biographique celle de méthode idiographique. Ce dernier terme « renvoie à la singularité et à la chose dans son idiosyncrasie» (p. 195) et aurait comme avantage de « déjouer

2. Igor Kopytoff, « The Cultural Biography of Things : commoditization as Process », dans Arjun Appadurai (dir.), The Social Life of Things : Commodities in Cultural Perspective, Cambridge, Cambridge University Press, 1986, p. 64-91. 
la critique linguistique suscitée par le bio-graphique dont le préfixe renvoie à une vie organique qui s'adapte mal au domaine de la matérialité. » (p. 196)

$\mathrm{Au}$ terme de cet essai très personnel dont il assume le caractère exploratoire et parfois provocant, Bonnot résume sa position : «si finalement l'objet ne peut pas être considéré comme une donnée plus objective pour l'anthropologie qu'un rituel ou un discours, c'est tout simplement parce qu'un anthropologue ne peut étudier l'objet que dans le cadre d'un système relationnel dans lequel il prend place. » (p. 199) L'attachement aux choses est un ouvrage riche, dense, bien structuré et s'appuyant sur une imposante bibliographie qui intègre les travaux de chercheurs européens et nordaméricains, francophones et anglophones. L'auteur n'hésite pas à mêler ses expériences de terrain, à revisiter certains concepts et à se soumettre à une auto-évaluation constante de ses manières de faire. Force est de constater que Thierry Bonnot vise juste en offrant une contribution originale parsemée de nombreuses pistes stimulantes qui viennent enrichir la réflexion sur le rapport aux choses et la compréhension de «l'objet en société ».

\section{Laurence Provencher-St-Pierre}

Université du Québec à Montréal

Bourgault, André-Médard et François Gauthier (coll.). La Maison de mon père. Préface de Michel Lessard. Belœil, Qualigram, 2015, XIX-156 p. ISBN 978-2-9815539-0-4.

La maison de mon père dont il est question dans ce livre, c'est celle du maître-sculpteur Médard Bourgault (1897-1967) qui fut à l'origine du mouvement de sculpture sur bois en taille directe qui a fait la réputation de Saint-Jean-Port-Joli.

Angéline Saint-Pierre (1931-2017), sculpteure, chroniqueuse et historienne, avait une admiration sans bornes pour Médard Bourgault, qu'elle avait pu observer au travail alors qu'elle apprenait la sculpture sur bois avec André, frère de Médard. Devant la richesse de l'héritage du maître-sculpteur, elle réalise après son décès survenu le 21 septembre 1967 que, malgré une pléthore d'articles de revues et de journaux sur l'homme et son œuvre, on ne trouve aucun ouvrage de synthèse. Elle publie Médard Bourgault, sculpteur, aux Éditions Garneau de Québec en 1973, ouvrage réédité aux Éditions Fides en 1981 et aux Éditions La Plume d'Oie en 2000. S'inspirant de l'immense documentation du Fonds Médard Bourgault - déposé depuis au Centre d'archives de la Côte-du-Sud à La Pocatière et qui est composé d'un journal, d'un carnet de bord, et d'une volumineuse correspondance, ainsi que des lettres de Médard à Marius Barbeau aujourd'hui aux archives du Musée canadien de 